\title{
REVIEWS
}

\section{Moderate alcohol consumption and coronary heart disease: a review}

\author{
L M Hines, E B Rimm
}

Consumption of alcoholic beverages has been a prominent feature of many cultures since the ancient civilisations. Throughout history, the pros and cons of alcohol have been debated. Although the original debates stemmed from the public perception of intoxication, current research has convincingly demonstrated the permanent physiological damage due to prolonged overconsumption. However, the effect of "moderate" alcohol consumption on overall health remains controversial. The beneficial effects of alcohol on the cardiovascular system have been touted since the 18th century, but the bulk of scientific research has accumulated over the last few decades. The majority of the literature suggests that alcohol in moderation is beneficial on the cardiovascular system, and excess is detrimental to overall health. The deleterious effects of heavy alcohol consumption on health are numerous: aerodigestive cancers; haemorrhagic stroke and cardiomyopathy; hepatic cirrhosis; fetal alcohol syndrome; fatal car crashes, and suicides. However, these effects have not been documented among light to moderate drinkers.

Although the risks substantially outweigh the benefits for heavy consumption, the burden of coronary heart disease (CHD) has tipped the scale in favour of moderate alcohol consumption for overall health. In the US and many westernised countries, CHD is the leading cause of mortality. It has been estimated that if moderate alcohol consumption lowered risk by even $25 \%$, more deaths could hypothetically be prevented than caused by this exposure in westernised countries. ${ }^{1}$ However, the concern that promoting moderate alcohol consumption may lead to alcohol abuse among certain "at risk" individuals has muted the avocation of moderate alcohol consumption for health reasons.

\section{Epidemiological evidence}

Evidence to support the cardioprotective effects of alcohol consumption among humans is abundant and strikingly consistent. Over 100 observational studies (cross cultural, casecontrol, prospective cohort) and over 80 short term human metabolic studies suggest cardiovascular benefits for 1-2 alcoholic beverages per day. ${ }^{2}$ Although a long term, large clinical trial of alcohol consumption and CHD would be optimal to confirm a causal association, it may not be feasible or ethical to conduct. However, there is overwhelming evidence from several epidemiological studies to support a causal association.

\section{ECOLOGICAL STUDIES}

Early epidemiological evidence to suggest an inverse association between moderate alcohol consumption and CHD was derived from ecological studies. These studies observed the correlation between alcohol intake and CHD mortality across countries. Data used in these ecological studies are typically based on estimated averages obtained from government agencies that tax the import, export, and sales of alcohol. As a whole, the majority of ecological studies support a strong inverse association between wine consumption and CHD mortality, but not for beer or spirits. ${ }^{3}$ However, specific benefits of wine beyond beer and spirits have not been confirmed with more methodologically sound study designs.

Although ecological studies are suggestive, they are not confirmatory because of the inherent flaws in this study design. One major limitation of the ecological study is the inaccurate representation of an individual's typical intake. In some countries, a small proportion of the population may consume the majority of alcohol. Furthermore, alcohol sales data do not take into account the sale to visitors that would most likely vary by beverage and country. The most important limitation is the inability to account for other important factors associated with both alcohol consumption and CHD risk. For example, wine consumers in certain countries may have a higher socioeconomic status, which may provide better access to healthcare. Their lower risk of CHD could be attributed to better access to healthcare rather than alcohol consumption.

CASE-CONTROL AND COHORT STUDIES

Case-control and cohort studies are preferred to the ecological study because they focus on the individual, thus, they provide information on individual intake and account for other factors related to $\mathrm{CHD}$ more accurately. In general, case-control studies are fairly quick and easy to conduct, but the results are not as reliable due to the retrospective nature of the study design. In a typical case-control study, healthy individuals and individuals with CHD are asked to recall their alcohol consumption habits in the past. This can bias the results because prior knowledge of disease status could influence the reported level of alcohol consumption. Furthermore, it would be difficult to assess the effect of alcohol consumption 
on fatal CHD. There are several good casecontrol studies that indicate an inverse association; however, the most compelling evidence is based on large, prospective cohort studies. ${ }^{2-4}$ In a prospective study, information on alcohol consumption is collected from healthy individuals when the study is initiated, which minimises the susceptibility to biased recall of alcohol consumption. After several years of follow up, the association between baseline alcohol consumption and newly diagnosed CHD, both fatal and non-fatal, can be determined.

Several large scale cohort studies among different populations have reported an inverse association between moderate alcohol consumption and risk of CHD and/or CHD mortality. ${ }^{2-6}$ Taking into account other known CHD risk factors, an overall estimate from prospective studies is a $30 \%-40 \%$ reduction in risk of CHD for two drinks/day among men and one drink/day among women, with minimal additional benefit for higher consumption levels. Depending on the study and the beverage type, a drink has been defined as containing $10-15 \mathrm{~g}$ of alcohol. An inverse association between alcohol consumption and CHD risk and/or CHD mortality has also been observed in diverse populations, such as Yugoslavia, France, China, Japan, Denmark, and Great Britain. ${ }^{7-12}$ The reproducibility of this finding among populations that differ with respect to incidence of CHD and preference of beverage type refutes previous ecological studies suggesting that only wine is beneficial. ${ }^{3}$

The benefit of moderate alcohol consumption has been observed for both fatal and nonfatal CHD. Evidence suggests a U-shaped relationship between alcohol consumption level and $\mathrm{CHD}$, with estimates of the bottom of the curve ranging from $2-6$ drinks/day. ${ }^{24}$ Some have argued that the inverse association between moderate alcohol consumption and CHD may be partially attributed to a poorly defined reference group. ${ }^{13}$ For example, the reference group of non-drinkers may include heavy drinkers who deny alcohol intake or who have stopped drinking alcohol due to illness. In general, these health related abstainers have a higher rate of diabetes, hypertension, and chronic health conditions. Studies that excluded individuals who abstain for health reasons have found a similar reduction in risk, suggesting that the reduction is not attributed to this potential bias. ${ }^{14} 15$

\section{Experimental studies}

Epidemiological studies have consistently shown that moderate alcohol consumption is associated with reduced risk of CHD; however, the exact mechanism for this association has not been completely elucidated. Over 80 experimental studies on humans have examined cardiovascular effects of alcohol on lipids, coagulation factors, and other cardiovascular markers. ${ }^{216}$ The majority of experimental studies utilised a crossover study design in which biological markers are measured among participants during abstinence and while consuming a fixed amount of alcohol after a washout period. Since these are relatively short term studies (less than three months), they are useful for providing information on the effects of alcohol on biological predictors of CHD and not on the effect of alcohol on clinical events.

The pathophysiology of coronary heart disease is a complex, but relatively well understood process that can be considered in two phases: (1) factors that promote plaque formation, instability, and rupture and (2) factors that initiate and control the progression of thrombosis after rupture. Studies have shown that alcohol exerts its effects on a number of cardiovascular markers, suggesting that there are several mechanisms involved. Based on a meta-analysis of these experimental studies conducted by Rimm et al, the effect of alcohol consumption on risk of CHD is attributed to high density lipoproteins (HDL; 60\%), fibrinogen $(20 \%-30 \%)$, insulin $(5 \%-10 \%)$, and haemostatic factors $(0 \%-5 \%))^{2}{ }^{16}$ Observational data, simultaneously estimating the relationships between alcohol, HDL, and risk of $\mathrm{CHD}$, have also demonstrated that at least half of the beneficial effect is attributed to HDL. ${ }^{17} 18$ Although the protective effect of HDL is understood, the mechanism(s) by which alcohol raises HDL levels is not clear. Some have speculated that alcohol may have a direct effect on the liver; it may increase hepatic production and secretion of apolipoprotein and lipoprotein particles, increase triglyceride lipases, and decrease removal of circulating HDL. ${ }^{19}$

Early ecological data suggesting that wine was more beneficial than beer or spirits prompted many in vitro and in vivo studies of antioxidant and antiplatelet effects of wine, but substantial additional benefit has not been established. ${ }^{20-22}$ Rimm et al did not observe any appreciable difference of HDL levels according to beverage type, which is consistent with the evidence from prospective studies. ${ }^{16}$ Overall, experimental studies suggest that ethanol, rather than other constituents of alcoholic beverages, is responsible for the cardioprotective changes.

LIPIDS

The effect of alcohol consumption on HDL occurs after 1-2 weeks of daily consumption and remains unless alcohol consumption level is decreased. ${ }^{2}$ Early observations suggested that alcohol mainly raised levels HDL-3 particles, not HDL-2, but recent work has found that it increases both. ${ }^{23}$ Although epidemiological evidence suggests a U-shaped association between alcohol consumption and CHD risk, the relationship between the amount of alcohol consumed and HDL level is linear and extends beyond the range of moderate consumption. ${ }^{16}$ The meta-analysis conducted by Rimm et al estimated that $30 \mathrm{~g} /$ day of alcohol (slightly over 2 drinks/day) would raise HDL by $0.10 \mathrm{mmol} / 1$ $(4 \mathrm{mg} / \mathrm{dl}) .{ }^{16}$ Gender, beverage choice, or duration of study did not noticeably influence this estimate. Alcohol consumption has been shown to have a greater impact on HDL levels among subjects with baseline levels below 1.0 $\mathrm{mmol} / \mathrm{l}(40 \mathrm{mg} / \mathrm{dl}) .^{2}$ Based on observational data, a $0.10 \mathrm{mmol} / 1(4 \mathrm{mg} / \mathrm{dl})$ increase in HDL 
would be associated with a $17 \%$ reduction in risk of CHD. ${ }^{16}$

Although HDL accounts for the majority of the reduction in risk of CHD, the effects of alcohol extend beyond that of HDL. Both triglycerides and $\mathrm{Lp}(\mathrm{a})$, a form of low density lipoprotein containing apo(a), are often raised among individuals with CHD. ${ }^{25}{ }^{26}$ The limited number of studies conducted on the association between alcohol consumption and $\mathrm{Lp}(\mathrm{a})$ levels have failed to show a consistent relationship. ${ }^{19}$ Studies have found a relatively weak but consistent positive association between alcohol and triglyceride concentration which may attenuate the benefit of alcohol on risk of CHD. Based on the meta-analysis conducted by Rimm et al, $30 \mathrm{~g} /$ day of alcohol would increase the triglyceride level by an estimated $0.06 \mathrm{mmol} / 1(5.69 \mathrm{mg} / \mathrm{dl})$, resulting in a $1.9 \%$ increased risk of coronary heart disease. ${ }^{16}$ This minimal increase in risk is negligible relative to the substantial effect of alcohol consumption on HDL levels.

HAEMOSTATIC AND OTHER FACTORS

Although not as well described, several studies have also investigated the influence of alcohol on coagulation and thrombolytic processes. ${ }^{1627-31}$ Briefly, normal haemostasis is maintained through balance between coagulation and fibrinolytic proteins. Vascular injury results in platelet aggregates that contain fibrinogen/fibrin, which can ultimately generate a thrombin. Alcohol can alter levels and interactions of fibrinolytic proteins to assist in preventing thrombosis.

In 1979, Meade et al demonstrated a positive association between alcohol consumption and fibrinolytic activity based on clot lysis time. ${ }^{27}$ Subsequent to the development of assays to measure the various cardiovascular markers of fibrinolytic activity, in vitro and in vivo studies have shown that alcohol affects fibrinogen levels, plasminogen activators, and plasminogen inhibitors. ${ }^{28}$ The effects on lowering levels of fibrinogen are the best documented and may contribute to the cardioprotective effects of ethanol. Based on the same meta-analysis by Rimm et al, fibrinogen is estimated to account for $20 \%-30 \%$ of the CHD benefit attributed to moderate consumption. ${ }^{216}$ Approximately 30 $\mathrm{g}$ /day of alcohol decreases fibrinogen by an estimated $0.075 \mathrm{~g} / 1(7.5 \mathrm{mg} / 100 \mathrm{ml})$, which corresponds to a $12.5 \%$ reduction of $\mathrm{CHD}$ risk. ${ }^{16}$ Although not nearly as substantial as the role of HDL, fibrinogen appears to be the second biggest contributor to the reduction in CHD risk attributed to alcohol consumption.

Plasminogen activators and inhibitors are also involved in haemostasis. Tissue-type plasminogen activator (t-PA) and urokinase-type plasminogen activator (u-PA) prevent thrombosis by degrading the fibrin clot, while plasminogen activator inhibitor type 1 (PAI-1) promotes thrombosis by inhibiting these activators. Iso et al observed a positive correlation between alcohol consumption and both plasma t-PA and PAI-1 antigen levels. ${ }^{29}$ Ridker et al also found that moderate alcohol consumption was associated with t-PA antigen levels. ${ }^{30}$ Veenstra et al investigated the short term effects of moderate alcohol consumption and found a significant decrease in t-PA and increase in PAI-1 during the postprandial phase. ${ }^{31}$ In addition to fibrinogen, other haemostatic factors may play an important part in the development of CHD. However, the overall contribution of other haemostatic factors to the reduction in risk of CHD attributed to alcohol consumption appears to be small (less than 5\%). ${ }^{216}$ Alcohol consumption has also been shown to increase insulin sensitivity. ${ }^{32} 33$ Similarly, the contribution of the effect of alcohol on insulin sensitivity may only account for a small portion (5\%$10 \%$ ) of the benefit on CHD risk. ${ }^{216}$

The development and application of new technologies has provided more insight to the effect of alcohol on the heart. In vitro and in vivo studies demonstrate that alcohol exerts its actions through several pathways that involve biochemical and molecular mechanisms of the normal and pathophysiological state of the heart. In addition to the previously discussed cardiovascular markers, alcohol affects the autonomic control adrenergic signalling, signal transduction in the myocardium, myocardial metabolism, and myocardial contractility. ${ }^{34}$ In order to fully understand how alcohol protects against CHD, it is necessary to elucidate all these complex mechanisms. Overall, epidemiological and experimental studies suggest that alcohol consumption decreases fibrinogen levels. There is some evidence to suggest that alcohol affects other haemostatic factors; however, the results are not consistent, which could be partially attributed to differences in collection and measurement methods.

\section{Alcohol, genetics, and heart disease}

Another important source of variability, which was not accounted for in the majority of human studies, is genetic differences among the study subjects. Polymorphisms have been identified in CHD candidate genes, such as $\mathrm{t}-\mathrm{PA},{ }^{35}$ PAI- $1,{ }^{36}$ and $\beta$-fibrinogen. ${ }^{37}$ Deciphering the human genome sequence has demonstrated that many genes contain variants, some of which will have an affect on the expression or function of the protein. These genetic differences may modify the effect of alcohol consumption on risk of $\mathrm{CHD}$ resulting in population variability in the amount of benefit achieved from alcohol consumption. For example, a few studies have suggested that the effect of alcohol intake on cholesterol levels is modulated by variability at the $A P O E$ locus. ${ }^{38} 39$ Thus, it is important to account for both genetic and lifestyle differences to better understand the causes of common chronic diseases such as heart disease.

Genetic differences contribute to the variability in ethanol metabolic capacity among individuals, which may influence the effect of alcohol consumption on alcohol related diseases. The pharmacokinetics of ethanol metabolism have been well studied. Alcohol dehydrogenase $(\mathrm{ADH})$ and the mitochondrial form of aldehyde dehydrogenase (ALDH2) are the two liver enzymes that are mainly responsible 


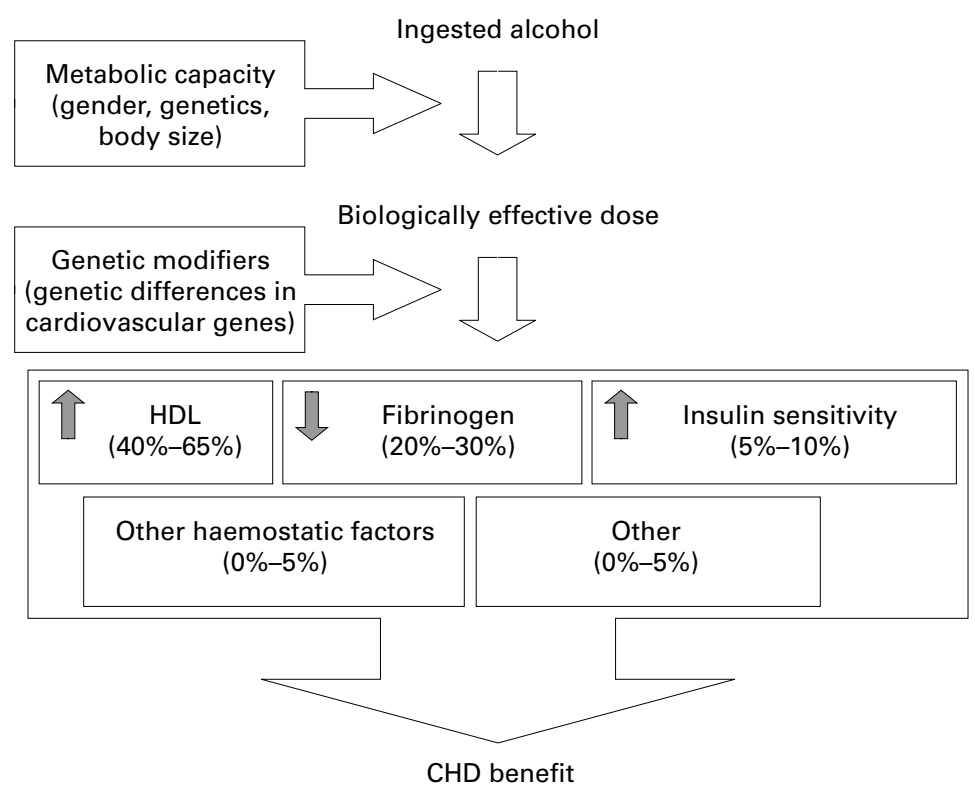

Figure 1 Mechanisms by which moderate alcohol consumption exerts its beneficial effects on the cardiovascular system $(C H D=$ coronary heart disease; $H D L=$ high density lipoprotein).

for alcohol metabolism. ${ }^{40}{ }^{41}$ The class I ADH enzymes, encoded by the $A D H 1, A D H 2$ and $A D H 3$ genes, are responsible for oxidising alcohol (ethanol) to acetaldehyde, and ALDH2 is responsible for acetaldehyde oxidation. Among the class I ADH genes, polymorphisms in $A D H 2$ and $A D H 3$ result in three different forms $\mathrm{ADH} 2$ enzyme $\left(\beta_{1}, \beta_{2}, \beta_{3}\right)$ and two different forms of the ADH3 enzyme $\left(\gamma_{1}, \gamma_{2}\right)$. These variants are known to produce enzymes with distinct kinetic properties. There are also two forms of the ALDH2 enzyme. The enzyme encoded by the variant $A L D H 2{ }^{\star} 2$ allele has very low activity, resulting in a flushing response due to the toxicity of acetaldehyde. The frequencies of these variants highly depend on the ethnicity of the population.

Among Caucasian populations, variant alleles are very rare at the $A D H 2$ and $A L D H 2$ locus, but common at the $A D H 3$ locus (approximately 55\% $\gamma_{1}: 45 \% \gamma_{2}$ ). This would suggest that the polymorphism at the $A D H 3$ locus would account for some of the variability in ethanol metabolic capacity among Caucasians. Among men in the Physicians' Health Study, moderate drinkers who were homozygous for the $A D H 3$ allele encoding for the slower enzyme $\left(\gamma_{2}\right)$ had a greater reduction in risk of heart disease compared with moderate drinkers who were homozygous for the $A D H 3$ allele encoding for the faster enzyme $\left(\gamma_{1}\right){ }^{42}$ Moderate drinkers who were homozygous for the $A D H 3$ allele encoding for the slower enzyme $\left(\gamma_{2}\right)$ also had the highest HDL levels; a similar observation was made among 325 postmenopausal women who were not taking postmenopausal hormones. Since the predominant function of $\mathrm{ADH} 3$ is to metabolise ethanol, this study strengthens the evidence that the reduction in risk of heart disease is attributed to the ethanol in alcoholic beverages.

Other contributors to the variability in alcohol metabolism are factors that can regulate the activity of the genes involved in alcohol metabolism. Glucocorticoids, growth hormone, and retinoids have been shown to induce $\mathrm{ADH}$ activity. ${ }^{43-45}$ To what extent that these factors contribute to population variability in alcohol metabolism needs to be determined.

To complicate the situation further, a number of in vitro studies have shown that ethanol influences gene expression in a variety of genes that may ultimately contribute to the development of heart disease, such as PPAR $a,{ }^{46}$ P4502E $1,{ }^{47} c-m y c,{ }^{48} t-P A,{ }^{49} u-P A,{ }^{49}$ and $P A I-1 .{ }^{50}$ Using cultured human endothelial cells, Grenett et al demonstrated that low doses of ethanol downregulate transcription of the PAI-1 gene, and upregulate transcription of both $t-P A$ and $u-P A$ gene expression..$^{50}$ This study indicates a biphasic increase (very rapid, $<30 \mathrm{~min}$ ) in fibrinolytic activity and a sustained long term effect ( $>12$ hours) involving increases in both $t-P A$ and $u-P A$ expression. Studies on the effects of alcohol consumption on gene regulation are limited, and further research in this area is warranted.

As described in this review, the mechanisms by which moderate alcohol consumption exerts its beneficial effects on the cardiovascular system are complex and not completely understood. To summarise, a simplified illustration of this complicated process is hypothesised in fig 1 .

\section{Drinking pattern}

Relatively few studies have assessed the significance of drinking pattern on risk of CHD. Based on experimental studies, there is some evidence to suggest that the regularity of consumption and the accompaniment of food can alter the effect of alcohol on various cardiovascular markers. ${ }^{51}{ }^{52}$ Epidemiological studies have shown that individuals consuming the entire week's allocation in a few days have either less or no benefit from alcohol consumption. ${ }^{53}{ }^{54}$ Sporadic heavy drinking may increase cardiovascular and stroke risks. ${ }^{54}$ There is no clear evidence to suggest that alcohol consumption with meals is different from alcohol consumption without meals on risk of CHD; however, there is some evidence to support that binge drinking is not beneficial.

\section{High risk populations}

Recent prospective studies indicate that high risk populations, men and women with diabetes, hypertension, or previous myocardial infarction, have a lower risk of subsequent CHD mortality with moderate alcohol consumption..$^{55-57}$ The limited evidence to suggest a beneficial effect of alcohol consumption on subsequent CHD among high risk populations is consistent, but limited to only a few studies. Thus, clinical advice advocating moderate alcohol consumption to patients with $\mathrm{CHD}$, diabetes, or hypertension should be considered cautiously.

\section{Gender differences}

Although lifetime mortality from CHD is similar between men and women, the disease primarily affects middle aged men and older 
women. Premenopausal women and postmenopausal women that use postmenopausal hormones have a substantially lower risk compared with men, suggesting a cardioprotective role of ovarian hormones. Experimental studies have shown that this is achieved through multiple mechanisms, such as increasing HDL levels, decreasing low density lipoprotein levels, and other vasculoprotective effects. ${ }^{58}$ There is some evidence to suggest that moderate alcohol consumption affects oestrogen levels. ${ }^{59}$ The effects of alcohol on the incidence of CHD could be associated with the effect of alcohol on oestrogen levels. Since the incidence of CHD in premenopausal women is virtually non-existent, it is difficult to assess the association between alcohol consumption on CHD risk in this population. However, epidemiological studies support a protective effect of alcohol consumption on CHD risk in postmenopausal women, and this benefit appears to be with lower levels of alcohol consumption (one drink/day). ${ }^{4}$ The gender difference is attributed to three main factors: (1) body size, (2) alcohol solubility, and (3) efficiency of alcohol metabolism. ${ }^{60}$ Alcohol is more soluble in body water than in body fat, and women typically have a higher proportion of body fat compared with men. Furthermore, the activity of $\mathrm{ADH}$ in the stomach and the first pass metabolism of alcohol before it enters systemic circulation are both lower in women. Thus, postmenopausal women achieve similar cardiovascular benefits from moderate alcohol consumption compared to men, but with lower levels of consumption.

\section{Conclusions}

The evidence to support a beneficial effect of alcohol consumption on cardiovascular health is overwhelming. However, is this evidence compelling enough to recommend moderate alcohol consumption from a public health perspective? Policies and legislation on alcohol consumption vary widely across countries. In some countries where CHD is the leading cause of mortality, the benefit of moderate alcohol consumption has been incorporated in the dietary guidelines. In the US, dietary guidelines on alcohol consumption are continuously modified to stay consistent with the scientific literature. ${ }^{61}$ Although the guidelines do not go as far as recommending alcohol to those who abstain, they do state that drinking in moderation may lower risk for $\mathrm{CHD}$, mainly among men over age 45 and women over 55, with moderation defined as one drink/day for women and two drinks/day for men. A typical drink is equivalent to one 12 ounce can of beer, one 5 ounce glass of wine, or 1.5 ounces of 80 proof spirits.

As demonstrated in this review, the mechanisms by which alcohol affects the cardiovascular system, as well as many other systems, is complex. A more complete understanding of these mechanisms will assist in making recommendations at the individual level, which would be the most effective means for promoting overall health. With any medical advice where there is risk and benefit, we should be cautious in framing guidelines for alcohol and

\section{Key messages}

- Moderate alcohol consumption, regardless of beverage type, reduces risk of coronary heart disease (CHD) among men and older women.

- There is strong evidence to support that ethanol, the main constituent in alcoholic beverages, is causally related to lower risk of CHD through changes in lipids and haemostatic factors.

- The effect of alcohol on high density lipoprotein levels accounts for the majority of the reduction in risk of CHD, however, the mechanisms by which alcohol exerts its protective effect on the cardiovascular system are very complex and not completely understood.

- Genetic factors modify the effect of alcohol consumption on risk of CHD, resulting in population variability in the amount of benefit achieved from alcohol consumption.

remember to keep them in the larger context of other lifestyle factors, such as exercise and diet, which we know can lower risk of chronic disease. Hennekens $\mathrm{CH}$. Alcohol and risk of coronary events. In:
Zahkari S, Wassef M, eds. Alcohol and the cardiovascular system. NIH Publication No 96-4133. Bethesda, MD: National tem. NIH Publication No 96-4133.

2 Rimm EB, Stampfer MJ. Alcohol abstinence: a risk factor for coronary artery disease. In: Braunwald E, ed. Heart disease update series. Philadelphia: W B Saunders, Vol 2, No 3, 2000: $1-10$.

3 Rimm EB, Klatsky A, Grobbee D, et al. Review of moderate alcohol consumption and reduced risk of coronary heart disease: is the effect due to beer, wine, or spirits? BMF 1996; 312:731-6.

4 Grobbee DE, Rimm EB, Keil U, et al. Alcohol and the cardiovascular system. In: MacDonald I, ed. Health issues related to alcohol consumption. Oxford: Blackwell Science, 1999: 125-79.

5 Sesso HD, Gaziano JM. Alcohol intake and cardiovascular morbidity and mortality. Curr Opin Nephrol Hypertens 1999; 8:353-7.

6 Gall N. Is wine good for your heart? A critical review. Postgrad Med f 2001;77:172-6.

7 Kozararevic D, McGee D, Vojvodic N, et al. Frequency of Kozararevic D, McGee D, Vojvodic N, et al. Frequency of
alcohol consumption and morbidity and mortality: the Yugoslavia Cardiovascular Disease Study. Lancet 1980;i: 613-6

8 Renaud SC, Gueguen R, Schenker J, et al. Alcohol and mortality in middle-aged men from eastern France. Epidemiology 1998;9:184-8.

9 Yuan JM, Ross RK, Gao YT, et al. Follow-up study of moderate alcohol intake and mortality among middle aged men in Shanghai, China. BMF 1997;314:18-23.

10 Kitamura A, Iso H, Sankai T, et al. Alcohol intake and premature coronary heart disease in urban Japanese men. Am $\mathcal{f}$ Epidemiol 1998;147:59-65.

11 Gronbaek M, Deis A, Sorensen TI, et al. Mortality associated with moderate intakes of wine, beer, or spirits. BMF 1995;310:1165-9.

12 Doll R, Peto R, Hall E, et al. Mortality in relation to consumption of alcohol: 13 years' observations on male consumption of alcohol: 13 years' ob
British doctors. BMF 1994;309:911-8.

13 Wannamethee SG, Shaper AG. Alcohol, coronary heart disease and stroke: an examination of the J-shaped curve. Neuroepidemiology 1998;17:288-95.

14 Rimm EB, Giovannucci EL, Willett WC, et al. Prospective study of alcohol consumption and risk of coronary disease in men. Lancet 1991;338:464-8.

15 Klatsky AL, Armstrong MA, Friedman GD. Risk of cardiovascular mortality in alcohol drinkers, ex-drinkers and nondrinkers. Am f Cardiol 1990;66:1237-42.

16 Rimm EB, Williams, P, Fosher K, et al. Moderate alcohol intake and lower risk of coronary heart disease: metaanalysis of effects on lipids and haemostatic factors. BMF 1999;19:1523-8.

17 Criqui MH, Cowan LD, Tyroler HA, et al. Lipoproteins as mediators for the effects of alcohol consumption and mediators for the effects of alcohol consumption and the Lipid Research Clinics Follow-up Study. Am f Epidemiol the Lipid Research 
18 Langer RD, Criqui MH, Reed DM. Lipoproteins and blood pressure as biological pathways for effect of moderate alco1992;85:910-5.

19 Dreon DM, Krauss RM. Alcohol, lipids, and lipoproteins. In: Zahkari S, Wassef M, eds. Alcohol and the cardiovascular system. NIH Publication No 96-4133. Bethesda, MD: National Institutes of Health, 1996: 369-91

20 Frankel EN, Kanner J, German JB, et al. Inhibition of oxidation of human low-density lipoprotein by phenolic substances in red wine. Lancet 1993;341:454-7.

21 Whitehead TP, Robinson D, Allaway S, et al. Effect of red wine ingestion on the antioxidant capacity of serum. Clin Chem 1995;41:32-5.

22 Bell JR, Donovan JL, Wong R, et al. (+)-Catechin in human plasma after ingestion of a single serving of reconstituted red wine. Am f Clin Nutr 2000;71:103-8.

23 Clevidence BA, Reichman ME, Judd JT, et al. Effects of alcohol consumption of lipoproteins of premenopausal 1995;15:179-84.

24 Gaziano JM, Buring JE, Breslow JL, et al. Moderate alcohol intake, increased levels of high-density lipoprotein and its subfractions, and decreased risk of myocardial infarction. $N$ Engl f Med 1993;329:1829-34.

25 Iso H, Naito Y, Sato S, et al. Serum triglycerides and risk of coronary heart disease among Japanese men and women. Am f Epidemiol 2001;153:490-9.

26 Rhoads GG, Dahlen G, Berg K, et al. Lp(a) lipoprotein as a risk factor for myocardial infarction. $\mathcal{F} A M A$ 1986;256: $2540-4$

27 Meade TW, Chakrabarti R, Haines AP, et al. Characteristics affecting fibrinolytic activity and plasma fibrinogen concentrations. BMF 1979;i:153-6.

28 Reeder VC, Aikens ML, Li XN, et al. Alcohol and the fibrinolytic system. In: Zahkari S, Wassef M, eds. Alcohol and the cardiovascular system. NIH Publication No 96-4133. Bethesda, MD: National Institutes of Health, 1996: 393-411.

29 Iso H, Folsom AR, Koike KA, et al. Antigens of tissue plasminogen activator and plasminogen activator inhibitor 1 : correlates in nonsmoking Japanese and Caucasian men and correlates in nonsmoking Japanese and Ca
women. Thromb Haemost 1993;70:475-80.

30 Ridker PM, Vaughan DE, Stampfer MJ, et al. Association of moderate alcohol consumption and plasma concentration of endogenous tissue-type plasminogen activator. $\mathcal{F} A M A$ 1994;272:929-33.

31 Veenstra J, Kluft C, Ockhuizen TH, et al. Effects of moderate alcohol consumption on platelet function, tissue-type plasminogen activator and plasminogen activator inhibitor. Thromb Haemost 1990;63:345-8.

32 Kiechl S, Willeit J, Poewe W, et al. Insulin sensitivity and regular alcohol consumption: large, prospective, cross sectional population study (Bruneck study). BMF 1996;313: 1040-4

33 Vitelli LL, Folsom AR, Shahar E, et al. Association of dietary composition with fasting serum insluin level: the dietary composition with fasting serum insluin level: the
ARIC study. Nutr Metab Cardiovasc Dis 1996;6:194-202.

34 Somlyo AP, Somlyo AV. Cellular and molecular mechanisms of smooth muscle. In: Zahkari S, Wassef M, eds. Alcohol and the cardiovascular system. NIH Publication No 96-4133.

Bethesda, MD: National Institutes of Health, 1996: 461-78.
Degen SJ, Rajput B, Reich E. The human tissue plasmino35 Degen SJ, Rajput B, Reich E. The human tissue plasn

gen activator gene. $\mathcal{F}$ Biol Chem 1986;261:6972-85. Dawson S, Hamsten A, Wiman B, et al. Genetic variation at the plasminogen activator inhibitor-1 locus is associated with altered levels of plasma plasminogen activat

inhibitor-1 activity. Arterioscler Thromb 1991;11:183-90.

37 Iso H, Folsom AR, Winkelmann JC, et al. Polymorphisms of the beta fibrinogen gene and plasma fibrinogen concentration in Caucasian and Japanese population samples. Thromb Haemost 1995;73:106-11.

38 Loktionov A, Scollen S, McKeown N, et al. Gene-nutrient interactions: dietary behaviour associated with high coronary heart disease risk particularly affects serum LDL cholesterol in apolipoprotein E 84 -carrying free-living indicholesterol in apolipoprotein E 84 -ca
viduals. Br $\mathcal{F}$ Nutr 2000;84:885-90.

39 Corella D, Tucker K, Lahoz C, et al. Alcohol drinking determines the effect of the APOE locus on LDL-cholesterol concentrations in men: the Framingham Offspring Study. Am $\mathcal{F}$ Clin Nutr 200;73:736-45.
40 Bosron WF, Lumeng L, Li TK. Genetic polymorphism of enzymes of alcohol metabolism and susceptibility to alcoholic liver disease. Mol Aspects Med 1988;10:147-58.

$41 \mathrm{Li} \mathrm{TK}$. Pharmacogenetics of responses to alcohol and genes that influence alcohol drinking. I Stud Alcohol 2000;61:512.

42 Hines LM, Stampfer MJ, Ma J, et al. Genetic variation in alcohol dehydrogenase and the beneficial effect of moderate alcohol consumption on myocardial infarction. $N$ Engl $\mathcal{F}$ Med 2001;344:549-55.

43 Dong Y, Poellinger L, Okret S, et al. Regulation of gene expression of class I alcohol dehydrogenase by glucocorticoids. Proc Natl Acad Sci U S A 1988;85:767-71.

44 Potter JJ, Yang VW, Mezey E. Influence of growth hormone on the synthesis of rat liver alcohol dehydrogenase in primary hepatocyte culture. Arch Biochem Biophys 1989;274: 548-55.

45 Duester G, Shean ML, McBride SM, et al. Retinoic acid response element in the human alcohol dehydrogenase gene ADH3: implications for regulation of retinoic acid synthesis. Mol Cell Biol 1991;11:1638-46.

46 Galli A, Pinaire J, Fischer M, et al. The transcriptional and DNA binding activity of perosixome proliferator-activated receptor $\alpha$ is inhibited by ethanol metabolism. $f$ Biol Chem $2001 ; 276: 68-75$.

47 Ingelman-Sundberg $\mathrm{M}$, Ronis $\mathrm{MJ}$, Lindros $\mathrm{KO}$, et al. Ethanol-inducible cytochrome P4502E1: regulation, enzymology and molecular biology. Alcohol Alcohol Suppl 1994;2: 131-9.

48 Paice AG, Hesketh JE, Richardson PJ, et al. The effect of starvation and ethanol on c-myc messenger RNA expression in the heart (abstract). Biochem Soc Trans 1996;24:212S.

49 Grenett HE, Aikens ML, Torres JA, et al. Ethanol transcriptionally upregulates t-PA and alcohol Clin Exp Res 1998 ; 22:849-53.

50 Grenett HE, Aikens ML, Tabengwa EM, et al. Ethanol downregulates transcription of the PAI- 1 gene in cultured human endothelial cells. Thromb Res 2000;97:247-55.

51 Hendriks HF, Veenstra J, Velthuis-te Wierik EJ, et al. Effect of moderate dose of alcohol with evening meal on fibrinolytic factors. BMF 1994;308:1003-6.

52 Numminen H, Kobayashi M, Uchiyama S, et al. Effects of alcohol and the evening meal on shear-induced platelet aggregation and urinary excretion of prostanoids. Alcohol Alcohol 2000;35:594-600.

53 Kannel WB. Alcohol and cardiovascular disease. Proc Nutr Soc 1988;47:99-110.

54 Kauhanen J, Kaplan GA, Goldberg DE, et al. Beer binging and mortality: results from the Kuopio ischaemic heart disease risk factor study, a prospective population based study. BMF 1997;315:846-51

55 Solomon CG, Hu FB, Stampfer MJ, et al. Alcohol intake and the risk of coronary heart disease mortality in persons with older-onset diabetes mellitus. Circulation 2000;102: 494-9.

56 Ajani UA, Gaziano JM, Lotufo PA, et al. Alcohol consumption and risk of coronary heart disease by diabetes status. Circulation 2000;102:500-5.

57 Muntwyler J, Hennekens CH, Buring JE, et al. Mortality and light to moderate alcohol consumption after myocardial infarction. Lancet 1998;352:1882-5.

58 Farhat MY, Myers AK, Ramwell PW. Alcohol, coronary alcohol and the cardiovascular system. NIH Publication No 96-4133. Bethesda, MD: National Institutes of Health, 1996: 647-61.

59 Purohit V. Moderate alcohol consumption and estrogen levels in postmenopausal women: a review. Alc Clin Exp Res 1998;22:994-7.

60 Meister KA, Whelan EM, Kava R. The health effects of moderate alcohol intake in humans: an epidemiologic review. Clin Rev Clin Lab Sci 2000;37:261-96.

61 US Departments of Agriculture and Health and Human Services. Nutrition and your health: dietary guidelines for Americans. 4th Ed. Washington, DC: US Government Printing Office, 1995. 